\title{
Componente Responsivo para Fachadas: Analise e Validação
} Responsive component for Facades: analysis and validation

\author{
Thales Barnuevo \\ Universidade de Brasília, Brasil \\ thalesbarnuevo@gmail.com \\ Lucas De Sordi \\ Universidade de Brasília, Brasil \\ lucasdesordi@gmail.com \\ Leandro Silva \\ Universidade de Brasília, Brasil \\ Leandro.mmqi@gmail.com
}

\author{
Neander Furtado Silva \\ Universidade de Brasília, Brasil \\ Neander.furtado@gmail.com \\ Francisco Leite Aviani \\ Universidade de Brasília, Brasil \\ cesco@unb.br
}

\begin{abstract}
The following text reports to the development of a climatic responsive component for facades programmed to respond to the movement of the sun as a way to reduce solar gain and glare into the interior ambient with the aim to reduce building's need for energy. This research is part of a methodology of tests to verify the relevance to develop, in the future, a real scale model into the Brazilian context.
\end{abstract}

Keywords: Ative facade; Dinamic; Responsive; Adaptive; Control.

\section{Introdução}

O controle do clima é um fator relevante na prática da arquitetura é desta maneira que Ghadiali (1959) dá sentido à arquitetura como a arte de projetar fazendo uso benéfico dos elementos da natureza para a criação de edifícios ambientalmente inteligentes e energeticamente eficientes.

Antes da introdução de sistemas mecânicos nas praticas de projeto, o desejado era 0 uso de tecnologias energeticamente passivas.

No Brasil, as condições do clima tropical sugerem a necessidade de controle da radiação solar estimulando o desenvolvimento de anteparos para fachadas e otimização do conforto térmico e luminoso dentro do ambiente construído. (Pinto et.al. 2015).

Com a introdução de sistemas de iluminação e de arcondicionado satisfazendo a necessidade de conforto ambiental no interior das edificações, arquitetos passaram a preocupar-se mais com a forma do edifício, o que contribuiu para o aumento do consumo energético (Bruna et.al. 2007).

Lopéz et.al. (2015) descreve que as características ambientais e climáticas de um determinado local são parâmetros variáveis, enquanto que aqueles relativos ao conforto, na arquitetura convencional, são estáticos, ou seja, temos edifícios com fachadas estáticas e equipamentos mecânicos dinâmicos para proporcionar conforto no ambiente interno.
Uma nova visão com relação às fachadas requer a separação do modelo convencional por uma nova tecnologia, com novas linguagens, promovendo o desenvolvimento e a implementação de técnicas dinâmicas e ambientalmente responsivas, com habilidade de controlar as mudanças do meio ambiente, como um passo necessário para a melhoria do desempenho energético nas edificações (IEA - ECBCS, 2009).

O termo responsivo, também descrito na bibliografia como reativo ou interativo, é entendido no contexto arquitetônico, como a capacidade de se adaptar e interagir dinamicamente com propriedades físicas e de desempenho energético de acordo com as variáveis e mudanças do ambiente interno e externo (Loonen et.al. 2013).

A presente pesquisa, de caráter transdisciplinar, assume a fachada como uma superfície interativa apoiado na definição de Loonen. Propomos a confecção de um Módulo Ativo Dinâmico e Responsivo (MADR) para controle da radiação e luz solar, visando a diminuição do consumo energético com sistemas de ventilação e refrigeração e iluminação artificial. O MADR é parte de uma metodologia de testes para verificar a pertinência de desenvolver, no futuro, um modelo piloto em escala real e subsequentemente, a construção de um produto comercial desenvolvido dentro do contexto brasileiro. 


\section{Problemática}

Fundamentadas na herança modernista do cobogó e do brise-soleil, tecnologias ativas para controle climático vem sendo aplicadas de forma crescente em várias partes do mundo. Gonçalo Castro Henriques (2015) defende que edifícios podem se adaptar melhor ao meio ambiente através de processos digitais integrados. No Brasil, ainda que esse seja pioneiro no desenvolvimento de anteparos passivos para controle da radiação e luz solar, não existem exemplos de fachadas com tecnologias ativas, dinâmicas e responsivas aplicadas na arquitetura.

\section{Hipótese}

Acreditamos que um módulo ativo, dinâmico e responsivo para controle da radiação solar nas fachadas e otimização da iluminação natural dentro do ambiente construído, como descreveremos neste artigo, constitui-se em hipótese promissora para o preenchimento da lacuna descrita em nossa problemática.

\section{Objetivo}

O objetivo deste trabalho propõe analisar, a partir da pesquisa em andamento, o desempenho de um Módulo Ativo, Dinâmico e Responsivo (MADR) como anteparo para fachadas, capaz de controlar a radiação solar na fachada e a incidência luminosa dentro do ambiente construído. Utilizando a metodologia de comunicação $1 / 0$ (input>processamento>output), o MADR visa à diminuição do consumo energético gerado pelo uso sistemas de ventilação e refrigeração e de iluminação artificial, reduzindo a carga térmica na fachada e regulando a entrada de luz solar dentro do ambiente construído. Produzido com uso de tecnologias de código aberto e componentes eletromecânicos de baixo custo, utilizando tecnologias de fabricação digital para a sua confecção.

\section{Procedimentos Metodológicos}

A pesquisa foi composta em cinco etapas, cada uma com um objetivo específico:

1. Investigação histórica de anteparos para controle térmico e luminoso em fachadas na arquitetura brasileira.

2. Estudo teórico sobre os principais sistemas dinâmicos e responsivos em uso e/ou em pesquisas.

3. Desenvolvimento da estrutura física do MADR.

4. Desenvolvimento e programação do sistema de comunicação e interatividade do MADR.

5. Avaliação do MADR em operação.

Detalhando cada etapa:

Etapa 1. Investigação histórica de anteparos para controle térmico e luminoso em fachadas na arquitetura brasileira.

Nesta etapa, nosso objetivo é identificar os principais anteparos para controle térmico e luminoso aplicado ao longo do processo historiográfico na arquitetura brasileira, como referência para a confecção de protótipo do MADR.

As primeiras referências que dispomos sobre anteparos de proteção térmica e luminosa na arquitetura brasileira provém de relatos de viajantes em expedições por tribos indígenas, como relatado por Elizabeth Agassiz apud Klüppel (2009) ao descrever a sensação de estar no interior de uma oca xavante: "a temperatura no interior da oca era agradável mesmo nas horas de maior rigor térmico. Toda feita de palha trançada permitia a circulação do ar, promovendo ventilação constante, além de filtrar a luz natural intensa".

Já no século XVI, a arquitetura colonial brasileira herdou o muxarabi, segundo diversos autores, vinculada à cultura árabe. Formado por um treliçado de madeira, este anteparo é definido por Klüppel (2009) como protelador térmico.

No início do século XIX, concedida a licença para a fabricação de vidro no Brasil, torna-se comum a introdução das vidraças e das janelas tipo guilhotina nas fachadas das construções daquela época. A introdução destes elementos gerou problemas de caráter térmico e luminoso com a entrada excessiva de luz natural, radiação solar direta e redução da ventilação em até $50 \%$ proporcionado pelas janelas em guilhotina (Klüppel, 2009). Para amenizar o desconforto foi introduzido o uso do de janelas com anteparo de veneziana.

$\mathrm{Na}$ primeira metade do século $\mathrm{XX}$, duas importantes contribuições de anteparos para controle térmico e luminoso foram desenvolvidas e largamente aplicadas na arquitetura moderna brasileira: o cobogó e o brise-soleil.

Segundo Vieira, Borba e Rodrigues apud Pinto et. al. (2015) o cobogó foi inventado no Recife, nos anos 20, concebido como um elemento pré-fabricado baseado numa retícula vazada sobre uma placa prismática de concreto, utilizado como elemento de composição para painéis verticais permitindo a passagem de ventilação e reduzindo a incidência de luz solar. O brise soleil, de acordo com Cunha (2011), Barber (2012) foi concebido por Le Corbusier para o projeto, não construído, da Maison Locative, na Argélia em 1933 e, reproduzido no Brasil pelos irmãos MMM Roberto no projeto para a Associação Brasileira de Imprensa - $A B I$, no Rio de Janeiro em 1936. Conforme proposto por Le Corbusier, a aplicação do brise soleil para a sede da $A B I$ consistiu de um anteparo formado por placas fixas de concreto dispostas diagonalmente, conforme ilustrado nas imagens (Figura $1 \mathrm{a}, \mathrm{b}$ ).

Oscar Niemeyer um ano após os irmãos MMM Roberto, no projeto Obra do Berço, no Rio de Janeiro (Figura 2c), faz uso do brise soleil de forma mais sofisticada, introduzindo o brise soleil móvel, de operação independente para cada andar, permitindo diferentes orientações no controle da radiação e luz solar (Barber 2012). 


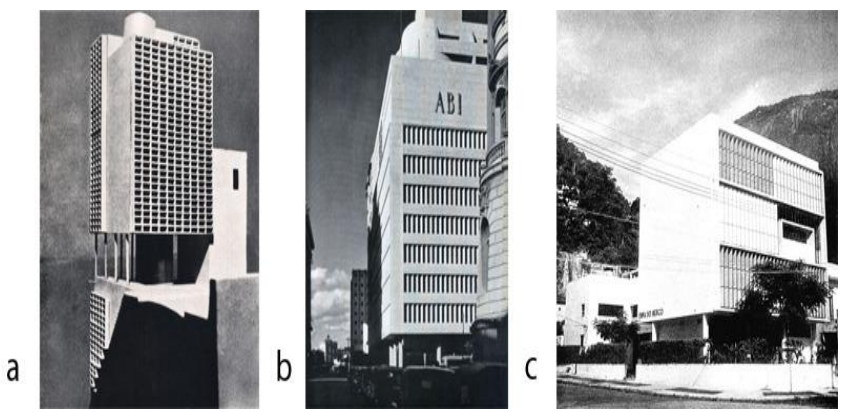

Figura 1: a) Maison Locative, 1933, Argélia - Le Corbusier. b) ABIAssociação Brasileira de Imprensa, 1936, Rio de Janeiro - MMM Roberto. c) Obra do Berço, 1937, Rio de Janeiro - Oscar Niemeyer. Fonte: www.issu.com (2012), www.archdaily.com.br (2013) e www. issuu.com (2012)

Etapa 2. Principais linhas teóricas em sistemas ativos, dinâmicos e responsivos ambientalmente inteligentes.

Em artigo escrito para o RIBA Journal na década de 80, intitulado $A$ wall for all seasons o arquiteto Mike Davies teorizou o princípio da polyvalent wall, que consistia em uma membrana com habilidade de absorver, refletir, filtrar e transferir energia do meio ambiente, composta por várias camadas cada qual cumprindo uma função responsiva específica como podemos ver na imagem (Figura 2a). De acordo com Davies, a polyvalent wall estaria em constante adaptação alterando seu estado conforme as condições do entorno, atuando como filtro em duas direções, interior e exterior.

Tomando a teoria de Davies como partida, classificamos em três as principais linhas de pesquisa para sistemas dinâmicos e responsivos, sendo estes: ativos, meteorosensitivos e por materiais inteligentes.

a) Sistema ativo. É o mais emblemático utilizado na arquitetura quando se refere a sistema climáticoresponsivo, na maioria dos casos, aplicado como anteparos para sombreamento. Na definição de Henriques (2015) são sistemas que geram sistemas capazes de responder em forma de movimento de acordo com os dados recebidos. Utiliza como metodologia de comunicação o sistema operacional $\mathrm{I} / \mathrm{O}$, requerendo de mecânica e componente eletromecânico complexo e, necessitando, ao menos, de uma fonte externa de energia para seu funcionamento.

Em termos espaciais, Moloney (2011) define o sistema ativo e dinâmico pelo movimento de três transformações geométricas básicas: translação, rotação e por deformação do material (expansão ou contração).

$\mathrm{Na}$ arquitetura, o uso deste sistema pode ser dividido pela sua escala de aplicação, podendo ser: estrutural, de superfície e de esquadria. $\mathrm{O}$ uso em escala estrutural ocorre quando há necessidade de abertura/fechamento de grandes vãos, a exemplo da cobertura do Museu do Amanhã, no Rio de Janeiro, projeto de Santiago Calatrava.
A aplicação em escala de superfície, aparte exemplos específicos como o edifício Aurora Place, em Sydney, Austrália, projeto de Renzo Piano, que utiliza de um sistema automatizado para abertura/fechamento das janelas; tem sido aplicado, na maioria dos casos, com finalidade em pesquisas acadêmicas com viés midiático escultural a exemplo do Super Cilia Surface, desenvolvido pelo grupo de pesquisa em Desenho Dinâmico do Instituto de Tecnologia de Massachusetts - MIT. A escala de esquadria é a mais comum aplicada à arquitetura, na grande maioria como anteparo de sombreamento, a exemplo do edifício Al Bahar Towers, em Abu Dahbi, projeto do escritório Ades Arquitetos, e que consiste em membranas instaladas no exterior da empena de vidro, que, em movimento de expansão/contração controla a incidência solar na fachada e regula a intensidade luminosa dentro do ambiente construído, como mostrado na Figura 2b.

Se por um lado o uso e aplicação do sistema ativo, dinâmico e responsivo representa o próximo passo na evolução das fachadas (Heusler, 2015) e, apesar das novas tecnologias disponíveis e do desenvolvimento de materiais inteligentes, ainda estamos distante da invenção de um componente de fachada plenamente adaptativo, responsivo e controlável (Anshuman apud Moloney, 2011).

b) Sistemas por material meteorosensitivo. Diferente do sistema ativo, não requer de mecânica e eletrônica complexa, nem fonte de energia elétrica. Caracteriza-se por ser um sistema dinâmico e responsivo passivo, que atua por princípio de deformação do material em resposta direta a uma condição atmosférica. Menges (2015) usa o termo "material máquina" para definir este sistema. A Figura 2c mostra a aplicação do sistema com material meteorosensitivo, utilizando material higroscópico no Pavilhão Meteoro-sensitivo: Hygro-skin, em Orleans, França.

Apesar de ser um sistema passivo que não requer fonte de energia, identificamos limitações que comprometem a sua aplicação como anteparo para fachadas. Uma delas é não permitir o controle humano do sistema, dependendo unicamente do desempenho dinâmico do próprio material, por outro lado, materiais meteorosensitivos aplicados como anteparos para controle climático em fachadas requer que sejam utilizados em forma de lâminas muito finas, o que compromete sua resistência e durabilidade quando exposto a intempérie.

c) Sistemas por materiais inteligentes. Uma das características deste sistema é a introdução do estudo da biologia na arquitetura através da biomimética. Para Oxman (2012) o estudo da biologia tem permitido transferir estratégias dos sistemas da natureza, implementando-as em soluções técnicas. Isto é possível graças às tecnologias disponíveis na atualidade que incluem: impressão 3D, avanços em tecnologias da computação e ao trabalho de equipes com conhecimento transdisciplinar, que tem viabilizando a criação de materiais inteligentes. Entendemos por materiais inteligentes aqueles 
desenvolvidos para responder a uma função programada ou pré-determinada. Oxman usa o termo "material ecology" para traduzir esta nova materialidade. Um exemplo da aplicação deste sistema é o projeto Dragon Fly, do MIT Media Lab - Instituto de Tecnologia de Massachusetts, ilustrado na Figura 2d, que consiste na fabricação de um novo material a base de quitina, um polissacarídeo encontrado no exoesqueleto de crustáceos, impresso por injeção em 3D em camadas com gramatura variada, ou seja, o mesmo material responde pela função estrutural e de vedação, apresentando diferentes características físicas do material como rigidez, elasticidade, opacidade $\mathrm{e}$ permeabilidade.

Este sistema apresenta um futuro promissor, porém, por estar em estágio inicial de pesquisas, inviabiliza sua aplicação na arquitetura no presente momento.
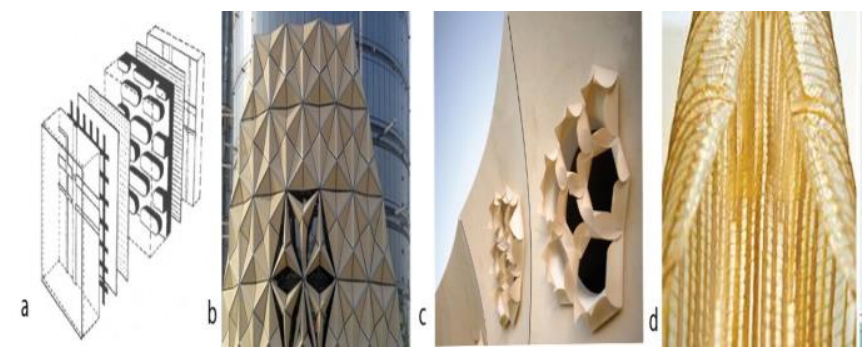

Figura 2: a) Polivalent Wall - Mike Davies. b) sistema ativo, dinâmico e responsivo. Al Bahar Towers, Abu Dahbi. - Ades Arquitetos. c) Sistema dinâmico e responsivo meteorosensitivo HygroSkin: Meteorosensitive Pavilion- Arq. Achim Menges. d) Sistemas de materiais inteligentes - Pavilhão Oceânico - Arq. Neri Oxman. Fonte: facadesconfidential.blogspot.com.br (2010), www.archdaily.com.br (2012), www.achimmenges.net (2013), http://matter.media.mit.edu (2015)

Além dos sistemas acima descritos, cabe ressaltar que existem sistemas que operam de forma mista, a exemplo do módulo ativo de ventilação para fachadas utilizando o sistema termoelétrico Peltier, uma pesquisa desenvolvida pelo Departamento de Edificações, Serviços e Estruturas da Faculdade de Arquitetura da Universidade de Navarra, Espanha (Ibáñez-Puy, 2015).

\section{Etapa 3. Estrutura física do protótipo MDR.}

O primeiro protótipo foi concebido para ser móvel, como parte de uma metodologia de testes para a implementação do modelo piloto em escala real, e subsequentemente, a construção de um produto comercial para fachadas.

A estrutura física do protótipo faz uma releitura dos principais anteparos para controle da radiação e luz solar aplicados em fachadas e identificados na revisão histórica da arquitetura brasileira.

O protótipo, conforme mostrado na Figura $3 a$ foi confeccionado utilizando tecnologia CAD, CAM.
Os painéis e a estrutura de suporte foram usinados em alumínio composto de $3 \mathrm{~mm}$ como visto na Figura 5 . As peças de fixação e rolamentos foram impressos em 3D utilizando filamento ABS.

O MADR proposto é formado por três painéis perfurados, posicionados verticalmente, sendo um painel fixo e dois móveis.

A operação de controle da incidência solar ocorre por meio da movimentação dos painéis. Quando os painéis estão alinhados, permitem maior entrada de luz, veja Figura 3b. Quando deslocados as aberturas se sobrepõem criando maior sombreamento conforme ilustrado na Figura 3c. Os painéis são alinhados ou sobrepostos através do deslocamento planar dos painéis móveis gerando diversas intensidades de sombreamento como visto na Figura 4.

A ação de abertura/fechamento é gerada pela tração de um servo motor instalado na parte inferior do painel central. No cabeçote do servo motor foi adaptada uma engrenagem fixa aos painéis móveis permitindo que estes se desloquem.
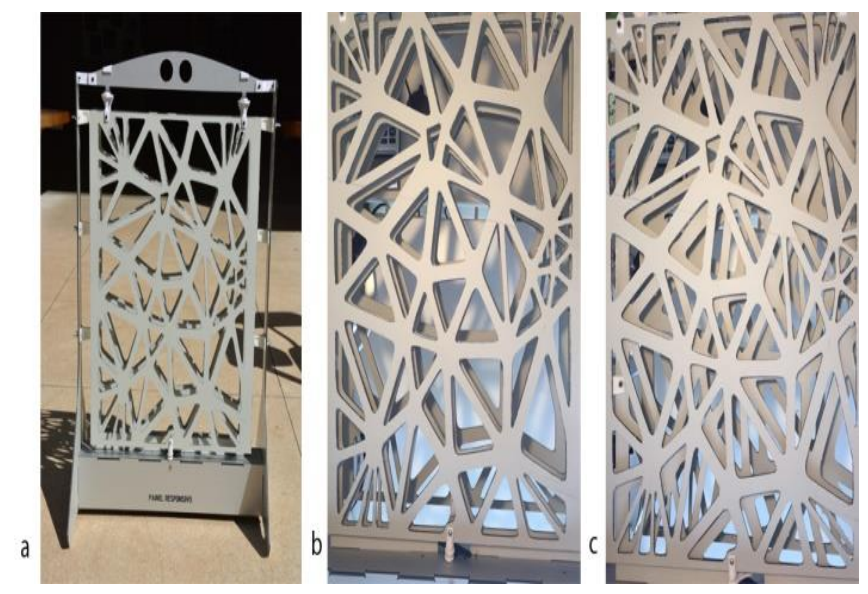

Figura 3. a) Protótipo Responsivo. b) painéis sobrepostos (maior entrada de luz). c) painéis deslocados (maior sombreamento). Fonte: Elaboração própria.
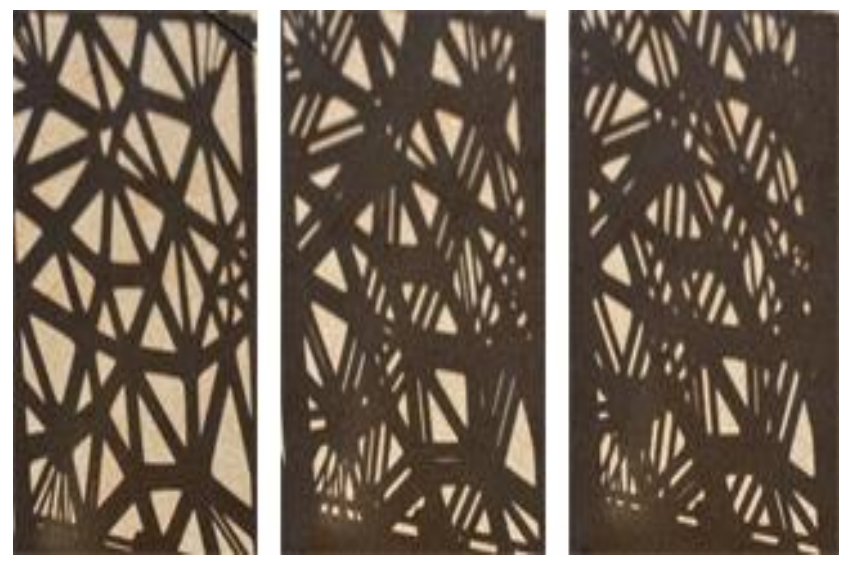

Figura 4. Escalas de sombreamento. Fonte: elaboração própria. 


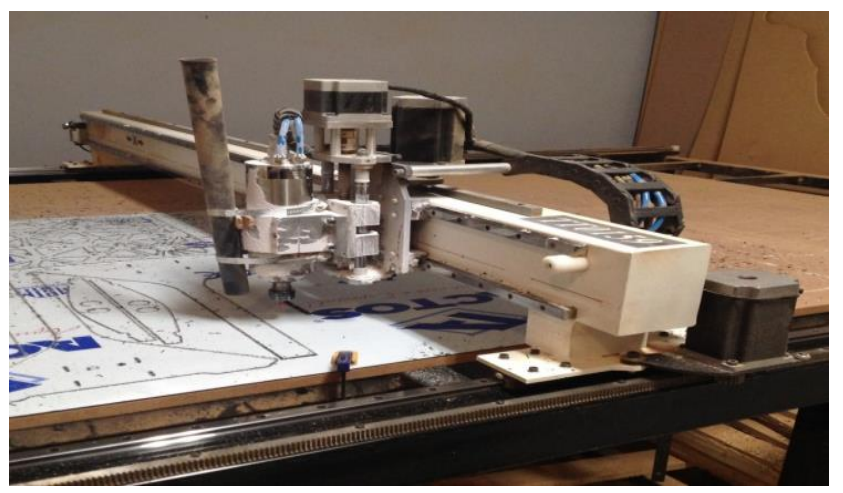

Figura 5. Peças sendo cortadas na CNC Router. Fonte: elaboração própria.

Etapa 4. Desenvolvimento do sistema interativo.

A opção pela confecção de um modelo reduzido, em dimensões e funcionalidade, foi pela necessidade de deslocamento para testes em diferentes contextos, assim como seria preciso que funcionasse de forma prática, investigado manualmente suas possibilidades. Sendo assim ele tem duas opções de controle, alterados por uma chave seletora: manual e automático.

No modo manual, o motor desloca os painéis em relação direta a um potenciômetro ligado ao sistema, podendo assim, simular as posições esperadas e mudar sua configuração instantaneamente. Quando a chave está posicionada no modo automático, o motor se altera suavemente seguindo a relação de luminosidade do ambiente.

O protótipo aqui proposto tem sido muito útil para mapear as variáveis postas ao sistema, como por exemplo, o ângulo mínimo e máximo que os painéis podem se mover, criando uma posição limite, fazendo que o motor não force um movimento que não pode fazer. Outro exemplo é o mapeamento do escopo máximo de luz de cada localidade, pois o sensor é capaz de sentir a luz do zero absoluto até uma quantidade de luz bastante intensa, que, no mundo real, especialmente nas cidades, raramente se encontra um escopo de variações tão grande.

Como sistema interativo, foi proposto uma metodologia que contemplava uma sensorização (input), um processamento de dados e tomada de decisão (processamento) e atuação (output), dando origem ao termo em inglês $\mathrm{I} / \mathrm{O}$ (Input > processamento > Output)

Os sensores usados foram sensor de luminosidade (LDS), medindo a luminosidade imediata do ambiente externo. Em um segundo momento, será colocado um segundo sensor LDS para criar um paralelo externo/interno de luminosidade. É esperado também a instalação de um sensor de temperatura.
Para o modelo piloto em escala real, que seria então fixo, a posição prevista do sol poderia ser salva no microcontrolador, gerando uma programação passiva da posição das pás de acordo com a posição do sol para aquela data, hora, localização e orientação da fachada do edifício. Também seria necessário um ajuste ativo do sistema, para adequar às condições meteorológicas no exato instante. Em um mesmo dia do ano, podem existir diferentes variações na intensidade da luz, de acordo com a formação das nuvens e/ou de dias chuvosos. Sendo assim, mesmo o sistema sabendo a posição exata do sol, ele poderia, por exemplo, abrir os painéis a poucos graus até encontrar uma situação de luz mais favorável.

Essas pequenas tomadas de decisão, comparações de luz e comandos de controle necessitam de um sistema que possa captar os impulsos. Esse sistema de processamento permite dar complexidade, criando comportamentos mais inteligentes, adaptativos e não lineares. Permitem também criar uma interface de controle e monitoramento do sistema.

Adotamos para este projeto o Arduino, um microcontrolador que contém portas configuráveis de controle, de leitura e comando eletrônico. O Arduino é ideal para o projeto, pois é de baixo custo, tem seu código aberto, é relativamente fácil de programar e é robusto o bastante para controlar sistemas de todos os tamanhos.

O Arduino é um projeto desenvolvido justamente para permitir que os designers possam criar sistemas eletrônicos interativos. Outra qualidade é a possibilidade de conecta-lo virtualmente a qualquer equipamento, permitindo criar interfaces de controle muito diferenciadas, como por celular com um módulo bluetooth, ou pela internet ligando-o a uma placa WI-FI, assim como, a fácil introdução de todo tipo de sensor no sistema eletrônico, como umidade, $\mathrm{CO}^{2}$, som, entre outros.

No protótipo em questão, foi usado o modelo mais comum atualmente, Arduino UNO r3, e no modelo real é previsto usar o Arduino Mega, por ter um ganho nas capacidades de processamento. Comercialmente seria produzida uma placa própria com o microprocessador do Arduino (ATMEGA) pois isso reduziria o custo do sistema.

O protótipo utiliza-se de um atuador, sendo esse um servo motor (SG-5010) leve, de torque máximo de $6,5 \mathrm{~kg} / \mathrm{cm}$. Esse motor é de fácil operação, tem feedback da sua posição para o sistema, é rápido e barato, ideal para um protótipo interativo. A Figura 6 mostra os componentes do sistema.

Já em um modelo de escala real, seria usado um motor de passo, existindo diversos modelos e potências no mercado, a escolha final vai depender das dimensões do sistema, ou seja, a necessidade de torque para mover todos os painéis. Esses motores de passo permitem mapear a posição do sistema, são mais lentos, porém detêm um torque superior. 
Seu preço é mais elevado, ainda assim economicamente viável.

É previsto para o sistema, sendo que esse tem uma leitura da luminosidade, introduzir uma placa de relés, ou um comando para um sistema de automação já existente, permitindo ligar e desligar a iluminação artificial e arcondicionado. Por exemplo, se ao fim do dia, mesmo com a abertura máxima dos painéis a luminosidade não for satisfatória em termos de conforto luminoso, para 0 trabalho em um escritório por exemplo, as luzes artificias podem ser ligadas gradualmente para acompanhar esse déficit.

Idealmente, esse sistema seria ligado a um sistema solar fotovoltaico, pois durante o dia se movimentaria por várias vezes, usando então da energia solar, mas ao fim do dia, sua função de controle de luz se torna menos ativo e as pás se moveriam para uma posição neutra, não precisando, então, de quase nenhuma energia para funcionar no modo de monitoramento.

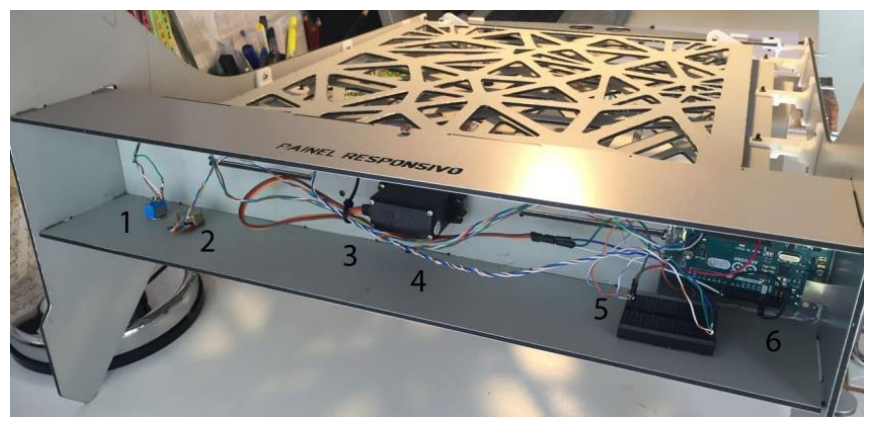

Figura 6. Sistema eletrônico: 1) switch. 2) potenciômetro. 3) sensor de luminosidade LDR - light dependente resistor. 4) servo motor SG-5010. 5) protoboard. 6) arduino Uno r3. Fonte: elaboração própria.

Etapa 5. Avaliação do sistema em operação.

O protótipo será colocado em operação para ser testado e avaliado. Dados resultantes dos testes ainda não estão disponíveis para ser divulgados neste artigo.

\section{Resultados}

Esta pesquisa visa avaliar o desempenho de um Módulo Ativo, Dinâmico e Responsivo - MADR, como um anteparo para controle da radiação solar em fachadas e regulagem da incidência luminosa dentro do ambiente construído. Ativado utilizando a metodologia de comunicação I/O (input>processamento>output), o MADR desempenha funções dinamicamente ajustáveis controlados indiretamente com ajuda de sensores. Este sistema processa a informação captada no ambiente físico transmitindo-a para os atuadores que respondem de acordo a uma ação programada. A opção pela construção de um protótipo móvel é parte de uma metodologia de testes para verificar a pertinência, no futuro, da construção de um produto comercial para fachadas inserido dentro do contexto brasileiro. Nesta fase da pesquisa foi fabricado

um protótipo com dimensão de $400 \times 500 \mathrm{~mm}$, composto por painéis de ACM de $3 \mathrm{~mm}$ e componentes eletromecânicos de baixo custo e utilizando software de código aberto para programação.

A escolha do material foi definida por ser largamente especificado como material de revestimento para fachadas na arquitetura. $\mathrm{O}$ objetivo principal nesta fase da pesquisa é avaliar o desempenho eletromecânico do sistema para fazer os ajustes necessários e produzir um modelo piloto em escala e situação física real.

\section{Discussão}

Uma agenda global para consumo eficiente de energia no ambiente construído tem aumentado o interesse por sistemas dinâmicos e responsivos como componentes para o controle da radiação e iluminação solar em fachadas. No Brasil, ainda que pioneiro no desenvolvimento de anteparos passivos como o cobogó e o brise soleil móvel, não existem exemplos de componentes ativos, dinâmicos e responsivos para fachadas.

Além de analisar o desempenho de um componente ativo, dinâmico e responsivo para fachada, este trabalho visa o intercâmbio de experiências com outras áreas de estudo dentro e fora da academia.

Originalmente o projeto é parte de uma pesquisa para a obtenção do título de Mestre em Arquitetura e Urbanismo e conta com a participação e colaboração de alunos da graduação da arquitetura e do desenho industrial, os quais foram introduzidos à problemática sobre 0 consumo energético nas edificações e na busca por alternativas de soluções com tecnologias ativas dinâmicas e responsivas para fachadas, como forma de contribuir à concepção de edifícios energeticamente mais eficientes.

\section{Referências}

IEA ECBCS Annex 44 Integrating Environmentally Responsive Elements in Buildings Expert Guide - Part 1 Responsive Building Concepts Editor: Per Heiselberg, Aalborg University, Denmark November 2009 www.civil.aau.dk/Annex44. p.44

Ghadiali, J.H.. (1959). Effect of Climate on Architectural Expression. In Seminar On Architecture, Edited by Achyut P. Kanvinde. New Delhi: Lalit Kala Akademi.

Pinto H., Queiroz N., Cardoso A., Sousa J.. (2015). O desenvolvimento de elementos de proteção de fachada responsivos: explorando o cobogó. SiGraDi 2015 - Project Information for Interaction. Pereira. A, Pupo. R (org.) XIX Congresso da Sociedade Ibero-Americana de Gráfica Digital - Volume 1 (pp. 519-527). Santa Catarina, 
Florianópolis. Universidade Federal de Santa Catarina. Bruna G., Fretin D., Maia R.. Do muxarabi ao brise-soleil: A

arquitetura brasileira ajusta-se ao clima. Cadernos de PósGraduação em Arquitetura e Urbanismo. São Paulo, SP.

Universidade Mackenzie. 2007. Disponível em: http://mackenzie.com.br.

López M., Rubio R., Martín S., Croxford B., Jackson R.. (2015). Ative materials for adaptative architectural envelopes based on plant adaptation principles. Journal of Façade Design and Engineering 3, 27-38.

Loonen R., Trčka D., Cóstola D., Hensen J.. (2013) Climate adaptative buildings shells: State-of-the-art and future challenges. Renewable and Sustainable Energy Reviews 25, 483-493.

Henriques G. (2015). Sitemas responsivos: relevância, estado da arte e desenvolvimentos. SIGraDi 2015 - Project Information for Interaction. Pereira. A, Pupo. R (org.) XIX Congresso da Sociedade Ibero-Americana de Gráfica Digital - Volume 1 (pp. 200-206). Santa Catarina, Florianópolis. Universidade Federal de Santa Catarina. Heusler W (2015). Latest developments in Building Skins. A new holistic approach. Advanced Building Skins. Englhardt O (Editor) International Conference on Buildings Envelope Design and Technology (pp. 1-11). Graz, Austria.

lbáñez-Puy M., Fernandez J., Gómez-Martín C., VidaurreArbizu M.. (2015) Development and construction of a thermoelectric active facade module. Journal of Façade Design and Engineering 3, 15-25.

Klüppel G. (2009). A casa e o clima: (Trans) Formações da arquitetura habitacional no Brasil (Século XII - século XIX). Salvador, BA. Universidade Federal da Bahia.

Cunha E. Brise-soleil: da estética à eficiência energética. Disponivel em: http://www.vitruvius.com.br.

Barber D. Le Corbusier, the brise-soleil, and the sócioclimatic Project of modern architecture, 1929-1963. 2012. Disponível em: https://issuu.com/thresholds/docs/thresholds40socio/28

Kirkegaard H., Parigi D. (2012). 'On Control Strategies for Responsive Architectural Structures: Proceedings: from spatial structures to space structures'. The International Association for Shell and Spacial Structures. In IASSAPCS, 2012.

Speck, T; Speck O. (2008). Process sequences in biomimetic research. Desing and Nature IV 3.

Oxman N. ,Soldevila L., Royo J.. (2015). Form follows flow: A material-driven computational workflow for digital fabrication on large-scale hierarchically structured objects. ACADIA 2015 - Computational Ecologies: Design in the Anthropocene. 